Mon. Not. R. Astron. Soc. 000,15(2014) Printed 25 August $2018 \quad$ (MN LATEX style file v2.2)

\title{
Modelling the 3D morphology and proper motions of the planetary nebula NGC 6302
}

\author{
L. Uscanga, ${ }^{1 \star}$ P. F. Velázquez, ${ }^{2}$ A. Esquivel, ${ }^{2}$ A. C. Raga,${ }^{2}$ P. Boumis,${ }^{1}$ \\ and J. Cantó, ${ }^{3}$ \\ ${ }^{1}$ Institute of Astronomy, Astrophysics, Space Applications and Remote Sensing, National Observatory of Athens, \\ 15236 Athens, Greece \\ ${ }^{2}$ Instituto de Ciencias Nucleares, UNAM, Apartado Postal 70-543, 04510 México, DF, Mexico \\ ${ }^{3}$ Instituto de Astronomía, UNAM, Apartado Postal 70-264, 04510 México, DF, Mexico
}

25 August 2018

\begin{abstract}
We present 3D hydrodynamical simulations of an isotropic fast wind interacting with a previously ejected toroidally-shaped slow wind in order to model both the observed morphology and the kinematics of the planetary nebula (PN) NGC 6302. This source, also known as the Butterfly nebula, presents one of the most complex morphologies ever observed in PNe. From our numerical simulations, we have obtained an intensity map for the $\mathrm{H} \alpha$ emission to make a comparison with the Hubble Space Telescope (HST) observations of this object. We have also carried out a proper motion (PM) study from our numerical results, in order to compare with previous observational studies. We have found that the two interacting stellar wind model reproduces well the morphology of NGC 6302, and while the PM in the models are similar to the observations, our results suggest that an acceleration mechanism is needed to explain the Hubble-type expansion found in $H S T$ observations.
\end{abstract}

Key words: methods: numerical - planetary nebulae: general - planetary nebulae: individual: NGC 6302

\section{INTRODUCTION}

Planetary nebulae (PNe) form around stars of low and intermediate mass $\left(\leqslant 8 \mathrm{M}_{\odot}\right)$ towards the end of their evolu' tion. The nebula originates from the interaction between a fairly dense $\left(\sim 10^{-5} \mathrm{M}_{\odot} \mathrm{yr}^{-1}\right)$ 'slow wind' $\left(\sim 10 \mathrm{~km} \mathrm{~s}^{-1}\right)$ lost by the star as a red giant on the asymptotic giant branch (AGB) with a tenuous $\left(\sim 10^{-8} \mathrm{M}_{\odot} \mathrm{yr}^{-1}\right)$ 'fast wind' $\left(\sim 1000 \mathrm{~km} \mathrm{~s}^{-1}\right)$ that follows after the AGB phase. The nebula material becomes ionized once the effective temperature of the central star, which evolves into a white dwarf, exceeds $25000 \mathrm{~K}$ (for a review see Balick \& Frank 2002).

NGC 6302 (PN G349.5+01.0) is a bipolar PN. Its complex and clumpy morphology shown in $\mathrm{H} \alpha$ and [N II] Hubble Space Telescope (HST) images (Szyszka, Zijlstra, \& Walsh 2011) can be roughly approximated by a bipolar shape with the two main lobes extending in the east-west direction. It presents a highly pinched waist which is characteristic for butterfly-shaped bipolar PNe (Balick \& Frank 2002). The central star of NGC 6302 was directly detected for the first time by Szyszka et al. (2009). It was partially obscured by a dense equatorial lane (Matsuura et al. 2005) and

* E-mail:lucero@astro.noa.gr molecular material observed in $\mathrm{CO}$ emission (Peretto et al. 2007; Dinh-V-Trung et al. 2008) that is tracing an expanding torus oriented in the north-south direction, approximately perpendicular to the optical nebula axis. The distance to this nebula was not known accurately. Gómez et al. (1989) gave a firm lower limit of $0.8 \pm 0.3 \mathrm{kpc}$ based on a radio expansion proper-motion ( $\mathrm{PM}$ ) measurement of the nebular core, and estimate a distance of $2.2 \pm 1.1 \mathrm{kpc}$ from measurements of pressure-broadening of radio recombination lines. Lately, Meaburn et al. (2008) determined an unambiguous distance of $1.17 \pm 0.14 \mathrm{kpc}$, derived from expansion PMs of 15 knots located at the northwestern lobe of the nebula. These PMs were measured comparing two groundbased images with a time separation of $\sim 51 \mathrm{yr}$, indicating a Hubble-type expansion. Hubble-type outflows are characterised by an expansion velocity that increases linearly with the distance to the central star. This type of outflow has been observed also in other objects, i.e., the nebula around the symbiotic system Hen 2-104 (Corradi et al. 2001), NGC 6537 (Corradi \& Schwarz 1993), Mz 3 (Redman et al. 2000), see Corradi (2004) for other examples.

Recently, Szyszka et al. (2011) measured the expansion PMs in NGC 6302, comparing two HST images in [N II] separated by 9.4 yr. The velocity field follows a Hubble law 
in agreement with the previous results of Meaburn et al. (2008). The PM vectors present a pattern mostly radial pointing back to the central source with a position close to the central star detected by Szyszka et al. (2009). Their results show that the lobes of NGC 6302 were ejected during a brief event $2250 \pm 35$ yr ago (in agreement with the result of Meaburn et al. 2008), and they find evidence for a subsequent acceleration that increased the velocity of the inner regions by $9.2 \mathrm{~km} \mathrm{~s}^{-1}$, possibly related to the onset of the ionization.

In this work, we investigate whether or not the morphological and kinematical characteristics of NGC 6302 can result from the interaction between an isotropic fast wind with a toroidally-shaped slow wind. We have also considered a clumpy structure for the slow wind, based on observations of AGB shells, which look like clumpy and filamentary shell structures (Cox et al. 2012; Steffen et al. 2013). The paper is organised as follows. In Section 2, we describe the basic assumptions and the initial conditions used in the numerical simulations. In Section 3, we present the results including synthetic $\mathrm{H} \alpha$ emission maps, simulated PMs of nebular knots, and their comparison with the observations. Finally, in Section 4, we discuss the implications of our results and give our conclusions.

\section{INITIAL CONDITIONS OF THE NUMERICAL SIMULATIONS}

Our simulations are based on the generalisation of the interacting stellar winds model (GISW) in which, an isotropic fast wind launched by a star expands into a previously ejected toroidally-shaped slow wind (Icke. Preston, \& Balick 1989; Mellema \& Frank 1995). We assumed that the slow wind has a density distribution with a high contrast between the equator and the pole, which is described by the following equation (given by Mellema 1995)

$\rho(r, \theta)=\rho_{0} g(\theta)\left(r_{0} / r\right)^{2}$,

with

$g(\theta)=1-\alpha\left[\frac{1-\exp \left(-2 \beta \cos ^{2} \theta\right)}{1-\exp (-2 \beta)}\right]$,

where $r$ is the distance from the central star and $\theta$ is the polar angle $\left(\theta=0^{\circ}\right.$ at the pole, and $90^{\circ}$ at the equator $)$. The parameter $\alpha$ determines the ratio between the value of the density at the equator and that at the pole, while $\beta$ determines the shape of the variation, and therefore that of the slow wind (see Mellema 1995). The value of $\rho_{0}$ can be calculated from the mass-loss rate $\dot{M}_{A G B}$ as:

$\rho_{0}=\dot{M}_{A G B} /\left(4 \pi r_{0}^{2} v_{A G B}\right)$,

where $v_{A G B}$ is the constant terminal velocity of the AGB wind and $r_{0}$ is the radius of the region where the stellar wind is imposed.

The 3D numerical simulations were performed with the YGUAzú hydrodynamical code (Raga, Navarro-González, \& Villagrán-Muniz 2000), which integrates the gas dynamical equations with a second-order accurate scheme (in time and space) using the 'flux-vector splitting' method of van Leer (1982) on a binary adaptive grid. A rate equation for neutral hydrogen is integrated together with the gas dynamics equations to include the radiative losses through a parametrized cooling function that depends on the density, temperature and hydrogen ionization fraction (Raga \& Reipurth 2004).

To model the NGC 6302 nebula, we have used a computational domain of $(1.5,1.5,3.0) \times 10^{18} \mathrm{~cm}$ along the $x$-, $y$-, and $z$-axes, respectively. Five refinement levels were allowed in the adaptive Cartesian grid, achieving a resolution of $5.9 \times 10^{15} \mathrm{~cm}$ at the finest level. For the initial condition we have filled the computational domain with the density distribution obtained from equation (1)-(3) with $\dot{M}_{A G B}$, $v_{A G B}$, and $r_{0}$ equal to $5 \times 10^{-4} \mathrm{M}_{\odot} \mathrm{yr}^{-1}, 15 \mathrm{~km} \mathrm{~s}^{-1}$, and $3.8 \times 10^{16} \mathrm{~cm}$, respectively. We have used $\alpha=0.999$ and $\beta=10$ in order to reproduce the observed morphology.

To simulate a 'clumpy' circumstellar medium (CSM), we have modulated the density by a fractal structure with a spectral index of $-11 / 3$, which is consistent with a turbulent interstellar medium (see Esquivel \& Lazarian 2005; Esquivel et al. 2003; Ossenkopf et al. 2006). This clumpy density is imposed on the initial condition and has fluctuations on the order of $20 \%$ of the mean density value. In this CSM, we start an isotropic and fast stellar wind with mass injection $\dot{M}_{f}$ of $10^{-7} \mathrm{M}_{\odot} \mathrm{yr}^{-1}$, and a velocity $v_{f}$ of $1800 \mathrm{~km} \mathrm{~s}^{-1}$.

\section{RESULTS}

\subsection{Synthetic $\mathrm{H} \alpha$ emission maps}

The 3D hydrodynamical simulations of two interacting winds were carried out with the setup given in Section 2 . The fast wind generates a shock wave which propagates into the surrounding CSM. A global bipolar morphology is generated and a similar size to the observed one is achieved after a time of $2000 \mathrm{yr}$, which is in agreement with the dynamical age given by Szyszka et al. (2011).

From the density and temperature distribution given by our 3D hydrodynamical simulations, we can perform synthetic $\mathrm{H} \alpha$ maps. In Fig. 1, we show the synthetic $\mathrm{H} \alpha$ emission maps obtained for the $x z-$ and $y z-$ projections (left and right panels of Fig. 1. respectively), using an angle $\phi=15^{\circ}$. This angle $\phi$ is the angle between the nebula axis (the $z$ axis of the computational domain) and the plane of the sky, in agreement with previous observational results (Peretto et al. 2007; Meaburn et al. 2008; Dinh-V-Trung et al. 2008). Fig. 1 shows a bipolar morphology for both projections, with a waist of $6 \times 10^{17} \mathrm{~cm}$, which has a similar size to the observed one ( $31^{\prime \prime}$ if a distance of $1.17 \mathrm{kpc}$ is considered). Furthermore, both panels display a filamentary and clumpy structure, which is a consequence of the interaction between the fast wind and the clumpy slow wind.

\subsection{Simulated PMs}

To calculate the PMs of the nebular knots we have re-started our simulation at an integration time of $2000 \mathrm{yr}$, and leave it to evolve by 10 additional yr. Then, we obtain the PMs of the knots found in the simulation using a cross-correlation 


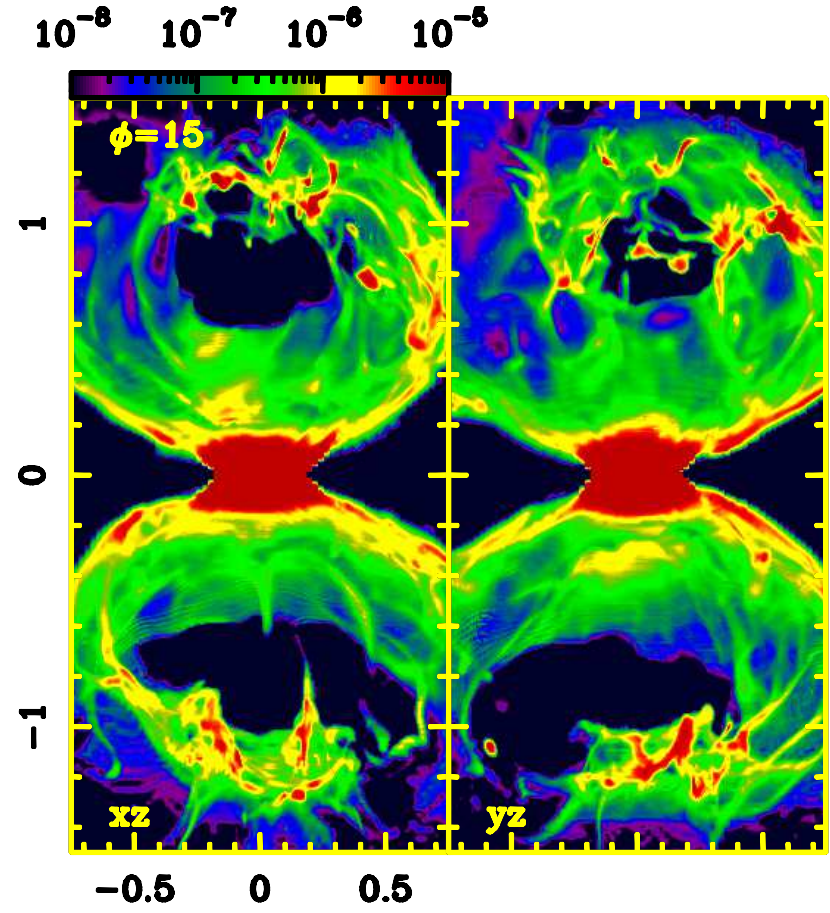

Figure 1. Synthetic $\mathrm{H} \alpha$ emission maps obtained at an integration time of $2000 \mathrm{yr}$ and for the $x z-$ and $y z-$ projections (left and right panel, respectively). The angle $\phi$ is the angle between the nebula axis (the $z$ - axis of the computational domain) and the plane of the sky. The vertical and horizontal axes are in units of $10^{18} \mathrm{~cm}$. The horizontal colour bar gives the $\mathrm{H} \alpha$ flux in units of erg $\mathrm{s}^{-1} \mathrm{~cm}^{-2} \mathrm{sr}^{-1}$.

method. We have used the "PM mapping" technique described by Raga et al. (2013), in which, the PMs from pair of images are derived by defining boxes including emitting knots and carrying out a cross-correlation function of the emission within the boxes. The PM is obtained from a fit to the peak of the cross-correlation function. This method has proven to be better than carrying out direct fits (e.g., a Gaussian or a two-dimensional paraboloid) to the observed emission features, because the cross-correlation functions, which are integrals of the emission within the chosen boxes, have higher signal-to-noise ratios than the images.

The cross-correlation boxes have a size of $30 \times 30$ pixels equivalent to $10 \times 10 \mathrm{arcsec}^{2}$. For a given box of size $L$, we first check whether or not the condition $f \geqslant f_{\text {min }}$ is satisfied in at least one pixel within the central inner box of size $L / 2$ (see appendix of Raga et al. 2013). Here $f$ is the $\mathrm{H} \alpha$ flux of the image and $f_{\min }$ is set equal to $10^{-7} \mathrm{erg} \mathrm{s}^{-1} \mathrm{~cm}^{-2} \mathrm{sr}^{-1}$. If this condition is met at least for a single pixel in each of the two epochs that are being analysed, the cross-correlation function (within the $L$-size box in the two images) and the PM are computed.

Fig. 2 shows the $y z-$ projection of the synthetic $\mathrm{H} \alpha$ map overlaid by white arrows which represent PM vectors calculated with the "PM mapping" technique. Interestingly, the PMs show deviations from the radial direction mostly for filamentary features in the outer regions of the outflow lobes. For a spatially extended filamentary structure, the PM perpendicular to the locus of the emission is well determined. While the component of the PM of the flow along the

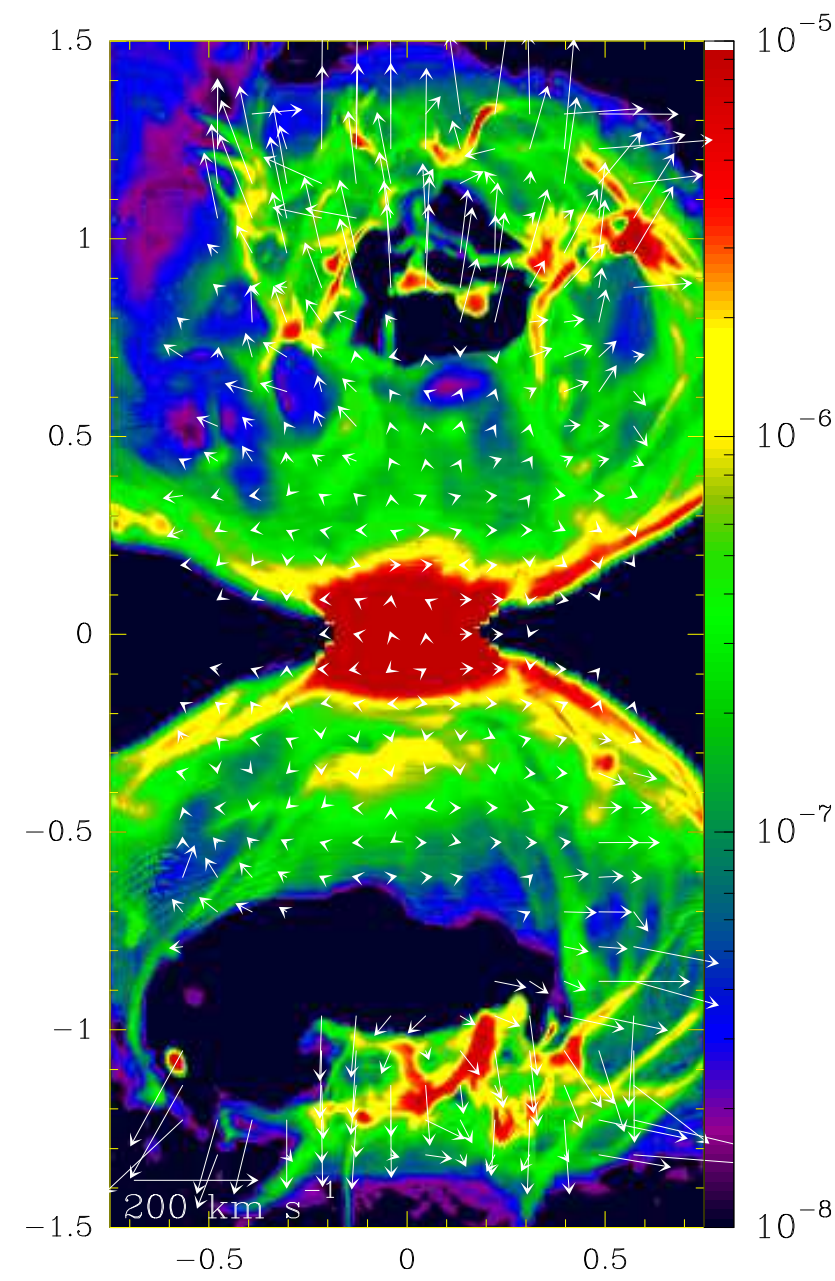

Figure 2. Relative $\mathrm{H} \alpha \mathrm{PM}$ map of NGC 6302. The nebula axis is oriented at $15^{\circ}$ with respect to the plane of the sky. The vertical and horizontal axes have the same dimensions as in Fig. 1] The vertical colour bar gives the $\mathrm{H} \alpha$ flux in units of $\mathrm{erg} \mathrm{s}^{-1} \mathrm{~cm}^{-2} \mathrm{sr}^{-1}$. The length of each arrow indicates the relative PM of the $\mathrm{H} \alpha$ emission knots computed in $10 \times 10 \operatorname{arcsec}^{2}$ boxes. The arrow at bottom left corresponds to a value of $200 \mathrm{~km} \mathrm{~s}^{-1}$.

filament is highly uncertain, resulting in a large dispersion of the PM vectors determined for filamentary features.

\subsection{Comparison between the simulated PMs and the observed PMs}

Fig. 3 shows PMs vs. distance to the central star plots for the top lobe (left panel) and bottom lobe (right panel). From this figure, we note that the more distant filaments and knots move faster than those located near the source. However, the PMs obtained from our model do not resemble the behaviour given by a Hubble-type expansion, such as found in the NGC 6302 observations (Szyszka et al. 2011). The Hubble-type expansion is represented in both panels of Fig. 3 by dashed lines. We can see from the Fig. 3 that many of the knots far from the source have velocities comparable to the observations, however we see a majority with velocities below the Hubble-type expansion. At the same time, the knots that are closer to the source $\left(\leqslant 5 \times 10^{17} \mathrm{~cm}\right)$, have a remarkably constant PM, well below the observations. This result sug- 

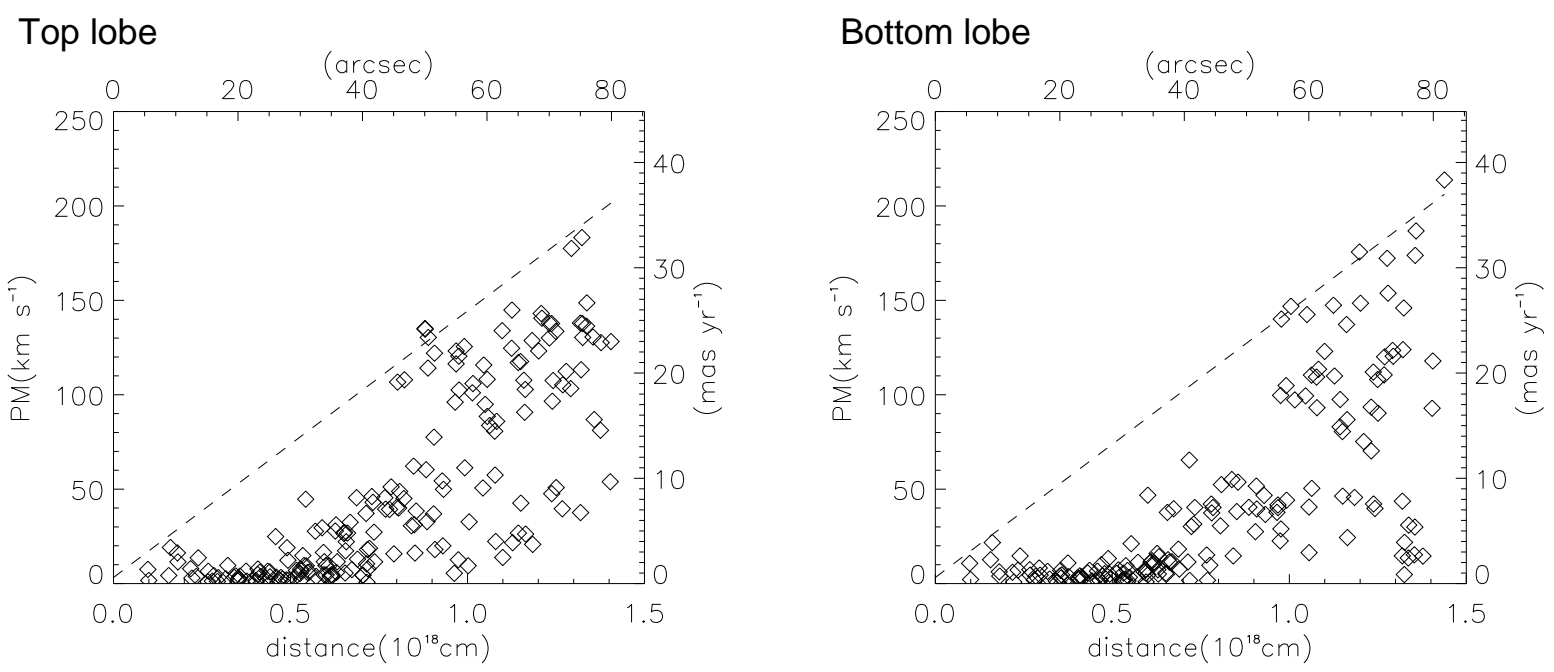

Figure 3. The PMs shown in Fig. 2 are plotted versus separation from the central source. The nebula axis is oriented at $15^{\circ}$ with respect to the plane of the sky. The left panel displays the PMs for $z>0$ (the top lobe of the synthetic nebula), while the right panel shows the PMs for $z<0$ (bottom lobe of the synthetic nebula). The diagonal line represents the linear fit of the observed PMs done by Szyszka et al. (2011).

gest that an additional acceleration mechanism is needed, particularly near the central star, which is consistent with the findings of Szyszka et al. (2011). A possible candidate for this acceleration mechanism is the ionizing flux from the central star, which would photoevaporate the clumps and push them through a rocket effect (see Esquivel \& Raga 2007). This, and any other acceleration mechanism is not included in our models, and will be pursued in a following work.

\section{DISCUSSION AND CONCLUSIONS}

We have carried out 3D hydrodynamical simulations of a two interacting-winds model, in order to explain both the global morphology and the tangential velocity of the PN NGC 6302. We reproduce the morphology and size of the nebula after an integration time of $2000 \mathrm{yr}$, which is similar to the dynamical time estimate given by Szyszka et al. (2011). We note the that it was necessary to use a high equator to pole density contrast of $\sim 1000$ to obtain the observed morphology.

A PM study was done on the synthetic $\mathrm{H} \alpha$ images during a time span of $10 \mathrm{yr}$ similar to the time span between observed images. The magnitude of the PMs as well as the separation from the central star are quantitatively similar, although the obtained PMs do not follow a Hubble-type expansion as the observations indicate. This suggests that an additional acceleration mechanism is acting, in particularly near the central star where the PM discrepancy is larger. A possible candidate for this mechanism is the rocket effect from the photoevaporation of the clumps, due to the radiation of the central star.

\section{ACKNOWLEDGMENTS}

We would like to dedicate this paper to the memory of our dear colleague Yolanda Gómez, who sadly passed away in
2012. She was also involved in this project. LU and PB are grateful to John Meaburn for his comments related to a very early draft version of this paper. We are thankful to our referee, Myfanwy Lloyd, for her valuable comments on the manuscript. LU acknowledges support from grant PE9-1160 of the Greek General Secretariat for Research and Technology in the framework of the program Support of Postdoctoral Researchers. PFV, ACR, JC, and AE acknowledge support from the CONACyT grants 167611, 167625, and UNAM DGAPA grant IG100214.

\section{REFERENCES}

Balick B., Frank A., 2002, ARA\&A, 40, 439

Corradi R. L. M., 2004, ASPC, 313, 148

Corradi R. L. M., Livio M., Balick B., Munari U., Schwarz

H. E., 2001, ApJ, 553, 211

Corradi R. L. M., Schwarz H. E., 1993, A\&A, 269, 462

Cox N. L. J., et al., 2012, A\&A, 537, A35

Dinh-V-Trung, Bujarrabal V., Castro-Carrizo A., Lim J.,

Kwok S., 2008, ApJ, 673, 934

Esquivel A., Lazarian A., 2005, ApJ, 631, 320

Esquivel A., Lazarian A., Pogosyan D., Cho J., 2003, MNRAS, 342, 325

Esquivel A., Raga A. C., 2007, MNRAS, 377, 383

Gómez Y., Rodríguez L. F., Moran J. M., Garay G., 1989, ApJ, 345, 862

Icke V., Preston H. L., Balick B., 1989, AJ, 97, 462

Matsuura M., Zijlstra A. A., Molster F. J., Waters L. B. F. M., Nomura H., Sahai R., Hoare M. G., 2005, MNRAS, 359, 383

Meaburn J., Lloyd M., Vaytet N. M. H., López J. A., 2008, MNRAS, 385, 269

Mellema G., 1995, MNRAS, 277, 173

Mellema G., Frank A., 1995, MNRAS, 273, 401

Ossenkopf V., Esquivel A., Lazarian A., Stutzki J., 2006, A\&A, 452, 223 
Peretto N., Fuller G., Zijlstra A., Patel N., 2007, A\&A, 473, 207

Raga A. C., Navarro-González R., Villagrán-Muniz M., 2000, RMxAA, 36, 67

Raga A. C., Noriega-Crespo A., Carey S. J., Arce H. G., 2013, AJ, 145, 28

Raga A. C., Reipurth B., 2004, RMxAA, 40, 15

Redman M. P., O'Connor J. A., Holloway A. J., Bryce M., Meaburn J., 2000, MNRAS, 312, L23

Steffen W., Koning N., Esquivel A., García-Segura G., García-Díaz M. T., López J. A., Magnor M., 2013, MNRAS, 436, 470

Szyszka C., Walsh J. R., Zijlstra A. A., Tsamis Y. G., 2009, ApJ, 707, L32

Szyszka C., Zijlstra A. A., Walsh J. R., 2011, MNRAS, 416, 715

van Leer B., 1982, Lecture Notes in Physics, 170, 507

This paper has been typeset from a $\mathrm{T}_{\mathrm{E}} \mathrm{X} / \mathrm{IAT}_{\mathrm{E}} \mathrm{X}$ file prepared by the author. 